\title{
Prevalence and risk indicators of non- carious cervical lesions in male footballers
}

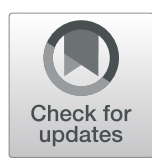

\author{
Tamea Lacerda Monteiro Medeiros', Sheila Cristina Almeida Neves Mutran', Daybelis González Espinosa', \\ Kelson do Carmo Freitas Faial ${ }^{2}$, Helder Henrique Costa Pinheiro ${ }^{1}$ and Roberta Souza D'Almeida Couto ${ }^{* *}$ (D)
}

\begin{abstract}
Background: Non-carious cervical lesions (NCCLs) have shown a significant incidence and prevalence and have been increasingly associated with people's lifestyles and youths. This cross-sectional study aimed to determine the prevalence of NCCLs in footballers and to address potential risk indicators.

Methods: Fourty-three male semi-professional footballers with an average of 27 years old completed a questionnaire and were subjected to intraoral examination in terms of cervical tooth wear, morphological characteristics of NCCLs, tooth sensitivity, occlusal/incisal wear, and malocclusion classification. Also, laboratory assays were performed to determine salivary parameters: flow rate, pH, buffer capacity, level of Ca (calcium), Na (sodium), and K (potassium) ions, and level of cortisol. The data obtained from the questionnaire and intraoral examinations were subjected to Chi-square and Poisson regression models while the data obtained from the laboratory assays were analyzed by using analysis of variance $(p<0.05)$.
\end{abstract}

Results: The prevalence of NCCLs was 39.5\%. The participants presented predominantly initial lesions with signs of mechanical stress. The daily training time was found as a significant risk indicator $(p=0.028)$. The multivariate analysis showed a significant difference in the variables daily training time $(p=0.023)$, lemon water intake while fasting ( $p=0.002)$, toothpaste type $(p=0.004)$, tooth sensitivity $(p=0.006)$; previous orthodontic treatment ( $p=$ $0.003)$, and occlusion type $(p=0.008)$. All participants presented normal salivary parameters and levels of cortisol.

Conclusion: The prevalence of NCCLs among footballers was remarkable. The premolars were the most affected teeth and presented symptoms/signs of initial lesions. The daily training time was a dominant risk indicator of NCCLs development. Footballers presented adequate salivary parameters and cortisol levels.

Keywords: Non-carious cervical lesions, Athlete, Saliva, Dentin

\section{Background}

Non-carious cervical lesions (NCCLs) are defined as the loss of dental hard tissue at the cementoenamel junction (CEJ) region without the action of microorganisms or inflammatory processes. These lesions vary from shallow saucer-shaped grooves to deep wedge-shaped defects and can occur sub- or supragingival at facial, lingual,

\footnotetext{
*Correspondence: rdalmeidacouto@gmail.com

${ }^{1}$ School of Dentistry, Federal University of Pará, Augusto Corrêa Street 937 odd side, Guamá, Belém, PA 66075110, Brazil

Full list of author information is available at the end of the article
}

and/or interproximal surfaces. Although this oral disease has a high incidence and prevalence regardless of age, gender, social, economic, and cultural conditions, it has been increasingly associated with people's lifestyles and youths [1-5]. NCCLs have a multifactorial etiology, including mechanical stress (tension), erosion (chemical degradation), and friction. Risk factors such as teeth clenching, premature or eccentric contacts, overbrushing, and acidic beverages intake modulate the evolution of NCCLs according to their intensity, duration, and frequency.

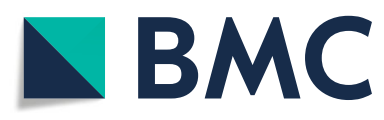

(c) The Author(s). 2020 Open Access This article is licensed under a Creative Commons Attribution 4.0 International License, which permits use, sharing, adaptation, distribution and reproduction in any medium or format, as long as you give appropriate credit to the original author(s) and the source, provide a link to the Creative Commons licence, and indicate if changes were made. The images or other third party material in this article are included in the article's Creative Commons licence, unless indicated otherwise in a credit line to the material. If material is not included in the article's Creative Commons licence and your intended use is not permitted by statutory regulation or exceeds the permitted use, you will need to obtain permission directly from the copyright holder. To view a copy of this licence, visit http://creativecommons.org/licenses/by/4.0/ The Creative Commons Public Domain Dedication waiver (http://creativecommons.org/publicdomain/zero/1.0/) applies to the data made available in this article, unless otherwise stated in a credit line to the data. 
The combination of factors seems relevant in the formation of NCCLs and mechanical stress is observed in most cases [6]. Individuals with lifestyles that enhance this peculiar interaction between tension, erosion, and friction are more vulnerable to NCCLs [7]. The intense physical exercises and high workloads that athletes are constantly submitted to increase the risk of body and dental trauma [8]. The fluid and electrolytes lost through sweat may cause dehydration and reduce the salivary flow rate. A dry mouth without the protection of saliva combined with acidic sports beverages intake significantly contributes to dental erosion [8-10]. Therefore, this study aimed to determine the prevalence of NCCLs in footballers and to address potential risk indicators.

\section{Methods}

\section{Participants and ethical aspects}

After receiving approval by the Ethics Committee of the Federal University of Pará (CAEE 86808318.2.0000.0018), male semi-professional footballers with at least 20 sound teeth (except for third molars) and not under orthodontic treatment were voluntarily enrolled in this cross-sectional study and signed a written informed consent form. Forty-five players of a football team represented the convenience sampling; therefore, considering an anticipated frequency of $50 \%$ at $95 \%$ confidence interval, the minimum sample size of 41 was determined with an open-source epidemiologic calculator (OpenEpi 3.01, SSPropor, Emory University, USA). Forty-three participants aged between 18 and 49 years (average of 27 years old) were evaluated from August to November 2018 at the School of Dentistry.

\section{Questionnaire}

The participants completed a questionnaire with regard to their name, gender, age, e-mail address, contact number, education level, occupation, daily training time, and years of a sports activity. The second part of the questionnaire addressed potential indicators of NCCLs development such as oral hygiene regime (toothbrush type, toothpaste, and brushing immediately after meals), medical conditions (dry mouth, gastroesophageal reflux, use of medication), diet (intake of acidic beverages), parafunctional habits (teeth clenching/grinding, nail or object biting), temporomandibular joint (TMJ) pain, tooth sensitivity, and previous orthodontic treatment (supplementary file).

\section{Intraoral examination}

One calibrated evaluator with intraevaluator reliability of 0.84 at the Kappa test performed the intraoral examinations at the School of Dentistry by using mirrors, periodontal, and exploratory probes. The cervical third of each tooth was measured with a periodontal probe to determine the tooth wear index (TWI) of Smith \& Knight modified by Soares \& Grippo [11]: $0=$ absence of NCCL; 1 = shallow NCCL with $<1 \mathrm{~mm}$ depth; $2=$ moderate NCCL with $1-2 \mathrm{~mm}$ depth; $3=$ deep NCCL with > $2 \mathrm{~mm}$ depth; and $\mathrm{R}=$ restoration, darkened surface, fracture, caries or calculus.

The morphological characteristics of NCCLs were classified by a) shape (rounded, wedged, or mixed), considering the angle formed at the pulp wall of the lesion; b) depth (shallow, moderate, and deep), in accordance with the cervical TWI criteria; c) anatomic localization (coronal or apical to the CEJ); d) clinical localization (supra- or subgingival), considering the relation between the apical margin of the lesion and the gingival margin and also the gingival margins of adjacent teeth as parameters; e) dentine aspect (with or without sclerotic dentin); and f) texture (smooth or rough).

The sensitivity level of each tooth was evaluated by using tactile and cold air stimuli. Tactile sensitivity was assessed using a cross-shaped exploratory probe to scratch the tooth surface from apical to occlusal/incisal and from mesial to distal directions. The evaporative stimuli were assessed through a blast of cold air from a triple syringe ( $40 \mathrm{psi}$ of pressure) applied approximately $2 \mathrm{~mm}$ from and perpendicular to the tooth surface. The participants were asked to qualify their sensitivity by using a modified visual analog scale (VAS): $0=$ no pain; $10=$ most severe pain .

In addition, the teeth occlusal surfaces were photographed and the dental arches were digitalized at maximum intercuspation with aid of an intraoral scanner (TRIOS ${ }^{\bullet}$ Pod, 3Shape, Copenhagen, Denmark). The images were exported to an orthodontics software (OrthoAnalyzer $^{\mathrm{m}}$ 3D, 3Shape Medical, Copenhagen, Denmark) and the occlusal/incisal wear of each tooth was classified in accordance with Mockers and modified by Vieira et al. [12]: $0=$ no wear; stage $1=$ enamel wear; stage 2 = dentin wear (the occlusal surface with more enamel than dentin; stage $3=$ dentin wear (the occlusal surface with more dentin than enamel); or stage $4=$ advanced wear stage (either close to or with exposure of the pulp chamber). The bite patterns were classified as Angle Class I, II, and III malocclusions; in addition, alterations such as open-, under-, over-, and crossbite were evaluated [13].

\section{Laboratory assays}

Two saliva samples of each participant were collected in the morning. The first sample was collected with the aid of a specific swab (Salivette tube ${ }^{\bullet}$ Cortisol Sarstedt AG \& Co., Nümbrecht, Germany). The level of salivary cortisol was determined through an electrochemiluminescence immunoassay (Cobas e411, Roche Diagnostics 
International, Basel, Switzerland) at a private laboratory (Paulo C. Azevedo, Belém, Brazil). Samples with concentrations below $20.3 \mathrm{nmol} / \mathrm{l}$ were considered normal.

Prior to the second sample collection, the participants chewed a piece of rubber tourniquet for $1 \mathrm{~min}$ and discarded the saliva; thus, the participants continued chewing and spit the saliva into a Falcon sterile tube every 1 -min interval during $5 \mathrm{~min}$. The second samples were used to determine the salivary parameters at the Laboratory of Biochemistry of the School of Pharmacy of the Federal University of Pará.

Salivary flow rates higher or equal to $0.7 \mathrm{ml} / \mathrm{min}$ were considered regular while values lower than $0.7 \mathrm{ml} / \mathrm{min}$ determined hyposalivation in accordance with the criteria established by Thylstrup \& Fejerskov [14]. The pH of saliva was measured with a $\mathrm{pH}$ meter (Thermo Scientific, South Logan, Utah, USA) calibrated with standardized solutions of $\mathrm{pH} \mathrm{4,} \mathrm{7,} \mathrm{and} 10$.

The saliva buffering capacity was assessed by actively mixing $1 \mathrm{ml}$ of saliva to $3 \mathrm{ml}$ of $0.005 \%$ hydrochloric acid. After $2 \mathrm{~min}$, the recipient was uncovered for 10 min for the evaporation of carbon gas, and the salivary $\mathrm{pH}$ was measured with the $\mathrm{pH}$ meter. In accordance with Krasse [15], values above or equal to and below 5.5 were classified as regular and low buffering capacity, respectively.

Samples containing $0.5 \mathrm{ml}$ of saliva and $0.5 \mathrm{ml}$ of $10 \%$ nitric were prepared, sieved with filter paper, and diluted in 1:10 deionized water. At the Evandro Chagas National Infectology Institute (Belém, Brazil), $\mathrm{Ca}, \mathrm{Na}$, and $\mathrm{K}$ ions were quantified by using an inductively-coupled plasma optical emission spectrometer (ICP-OES) (Varian VistaMPX CCD Simultaneous, Varian Analytical Instruments, Mulgrave, Australia), under axial configuration and equipped with an automatic sampling system (SPS-5 Varian Spectrophotometer AutoSampler, Varian Analytical Instruments) and specific software (ICP Expert Vista, Varian Analytical Instruments).

\section{Statistical analysis}

The data obtained from the questionnaire and intraoral examinations were subjected to descriptive analysis, bivariate analysis through the Chi-square test, bivariate and multivariate Poisson regression models (SPSS 2.0, IBM Corp., Chicago, USA). The data obtained from the laboratory assays were analyzed by using analysis of variance (BioEstat 5.0, Mamirauá Institute, Belém, Brazil). A significance level of $95 \%(p<0.05)$ was used for all analyses.

\section{Results}

Most of the participants had a high level of education (58.1\%), more than two years of a sports activity (62.8\%), and more than $1 \mathrm{~h}$ of daily training (55.8\%). Only $11.6 \%$ of the participants reported football as their exclusive occupation. Approximately $79 \%$ of the participants reported to use conventional toothpaste, $27.9 \%$ used to brush their teeth immediately after meals, $30.2 \%$ reported to have dry mouth, $11.6 \%$ reported gastroesophageal reflux, 23.2\% used medication, 9.3\% reported to drink lemon water while fasting, $74.4 \%$ reported parafunctional habits, $27.9 \%$ reported TMJ pain, $79 \%$ presented tooth sensitivity and $30.2 \%$ reported previous orthodontic treatment. The majority of the participants (81.4\%) presented Angle Class I malocclusion and 41.9\% presented an altered bite. The NCCLs were diagnosed in $39.5 \%$ of the participants. The footballers that reported up to $1 \mathrm{~h}$ of daily training had a significantly higher prevalence of NCCLs than those that trained for more than $1 \mathrm{~h}$ per day $(p=0.028)$ (Table 1$)$.

The bivariate analysis revealed that the footballers that trained up to $1 \mathrm{~h}$ per day (PR 2.32 [1.05-5.11]; $p=0.038$ ) and those who consumed lemon water while fasting (PR 2.5 [1.67-3.75]; $p<0.001)$ showed a significant association with the development of NCCLs. The multivariate analysis with adjusted values revealed a significant association between NCCLs and up to $1 \mathrm{~h}$ of daily training (PR 3.35 [1.18-9.49]; $p=0.023$ ), lemon water intake while fasting (PR 8.10 [2.19-29.90]; $p=0.002$ ), use of desensitizing toothpaste (PR 5.30 [1.63-16.96]; $p=0.004$ ), presence of tooth sensitivity (PR 2.53 [1.31-4.88]; $p=$ 0.006 ), and previous orthodontic treatment (PR 3.11 [1.48-6.50]; $p=0.003$ ) (Table 2).

Occlusal/incisal wear was more frequently observed in anterior teeth (39.7\%) and canines (29.1\%) followed by premolars (16.3\%) and molars (10.9\%). The same trend was observed for tooth sensitivity evaluations: anterior teeth (21-24.5\%), canines (24.4-23.3-\%), premolars (18.1-21.1\%), and molars (16.8-15.6\%) (Table 3).

NCCLs were diagnosed in $8 \%$ of the teeth examined (94 of 1167). These lesions were more frequently found in premolars (16.9\%), in the upper arch (8.8\%), and on the right side $(9.9 \%)$. The NCCLs were predominantly shallow $(<1 \mathrm{~mm}$ depth) and diagnosed in premolars (15.8\%). The lesions of moderate depth $(1-2 \mathrm{~mm})$ were only observed in premolars $(0.9 \%)$ and molars $(0.9 \%)$. Class V restorations were more frequently observed in molars (2\%) (Table 3).

Among the teeth with shallow NCCLs $(<1 \mathrm{~mm}), 83 \%$ did not present occlusal/incisal wear and $17 \%$ presented wear only in enamel. Occlusal/incisal wear was not observed in those teeth with moderate NCCL $(1-2 \mathrm{~mm}$ depth) (Table 4). Therefore, the relationship between occlusal/incisal tooth wear and NCCLs depth was not significant $(p=0.077)$.

The most frequent morphological characteristics of the NCCLs were: rounded shape (65\%), shallow depth (94\%), coronal to the CEJ (52\%), supragingival (52\%), 
Table 1 Descriptive and bivariate analysis of the questionnaire variables

\begin{tabular}{|c|c|c|c|c|}
\hline Variables & Total (\%) & $\mathrm{NCCL}+(\%)$ & NCCL- (\%) & $p$-value \\
\hline Education level & & & & 0.638 \\
\hline High School & $11(25.6)$ & $5(45.5)$ & $6(54.5)$ & \\
\hline University & $25(58.1)$ & $8(32)$ & $17(68)$ & \\
\hline Postgraduate & $7(16.3)$ & $4(57.1)$ & $3(42.9)$ & \\
\hline Years of a sports activity & & & & 0.834 \\
\hline Up to 2 years & $16(37.2)$ & $6(37.5)$ & $10(62.5)$ & \\
\hline More than 2 years & $27(62.8)$ & $11(40.7)$ & $16(59.3)$ & \\
\hline Daily training time & & & & $0.028^{*}$ \\
\hline Up to $1 \mathrm{~h}$ & $19(44.2)$ & $11(57.9)$ & $8(42.1)$ & \\
\hline More than $1 \mathrm{~h}$ & $24(55.8)$ & $6(25)$ & $18(75)$ & \\
\hline Occupation & & & & 0.293 \\
\hline Student & $7(16.3)$ & $3(42.9)$ & $4(57.1)$ & \\
\hline Worker & $18(41.9)$ & $8(44.4)$ & $10(55.6)$ & \\
\hline Student and worker & $13(30.2)$ & $6(46.2)$ & $7(53.8)$ & \\
\hline Without occupation & $5(11.6)$ & $0(0)$ & $5(100)$ & \\
\hline Toothpaste & & & & 0.483 \\
\hline Conventional & $34(79)$ & $12(35.3)$ & $22(64.7)$ & \\
\hline Desensitizing & $3(7)$ & $2(66.7)$ & $1(33.3)$ & \\
\hline Whitening & $6(14)$ & $3(50)$ & $3(50)$ & \\
\hline Brushing immediately after meals & & & & 0.383 \\
\hline No & $31(72.1)$ & $11(35.5)$ & $20(64.5)$ & \\
\hline Yes & $12(27.9)$ & $6(50)$ & $6(50)$ & \\
\hline Dry mouth & & & & 0.146 \\
\hline No & $30(69.8)$ & $14(46.7)$ & $16(53.3)$ & \\
\hline Yes & $13(30.2)$ & $3(23.1)$ & $10(76.9)$ & \\
\hline Gastroesophageal reflux & & & & 0.319 \\
\hline No & $38(88.4)$ & $14(36.8)$ & $24(63.2)$ & \\
\hline Yes & $5(11.6)$ & $3(60)$ & $2(40)$ & \\
\hline Medication & & & & 0.149 \\
\hline No & $33(76.8)$ & $15(45.5)$ & $18(54.5)$ & \\
\hline Yes & $10(23.2)$ & $2(20)$ & $8(80)$ & \\
\hline Lemon water intake while fasting & & & & 0.128 \\
\hline No & $39(90.7)$ & $14(35.9)$ & $25(64.1)$ & \\
\hline Yes & $4(9.3)$ & $3(75)$ & $1(25)$ & \\
\hline Parafunctional habits & & & & 0.642 \\
\hline No & $11(25.6)$ & $5(45.5)$ & $6(54.5)$ & \\
\hline Yes & $32(74.4)$ & $12(37.5)$ & $20(62.5)$ & \\
\hline TMJ pain & & & & 0.605 \\
\hline No & $31(72.1)$ & $13(41.9)$ & $18(58.1)$ & \\
\hline Yes & $12(27.9)$ & $4(33.3)$ & $8(66.7)$ & \\
\hline Tooth sensitivity & & & & 0.735 \\
\hline No & $9(21)$ & $4(44.4)$ & $5(55.6)$ & \\
\hline Yes & $34(79)$ & $13(38.2)$ & $21(61.8)$ & \\
\hline Previous orthodontic treatment & & & & 0.206 \\
\hline
\end{tabular}


Table 1 Descriptive and bivariate analysis of the questionnaire variables (Continued)

\begin{tabular}{|c|c|c|c|c|}
\hline Variables & Total (\%) & $\mathrm{NCCL}+(\%)$ & NCCL- (\%) & $p$-value \\
\hline No & $30(69.8)$ & $10(33.3)$ & $20(66.7)$ & \\
\hline Yes & $13(30.2)$ & $7(53.8)$ & $6(46.2)$ & \\
\hline Malocclusion & & & & 0.763 \\
\hline Angle Class I & 35 (81.4) & $14(40)$ & $21(60)$ & \\
\hline Angle Class II & $4(9.3)$ & $1(25)$ & $3(75)$ & \\
\hline Angle Class III & $4(9.3)$ & $2(50)$ & $2(50)$ & \\
\hline Bite alteration & & & & 0.576 \\
\hline No & $25(58.1)$ & $9(36)$ & $16(64)$ & \\
\hline Yes & $18(41.9)$ & $8(44.4)$ & 10 (55.6) & \\
\hline Total (\%) & $43(100)$ & 17 (39.5) & $26(60.5)$ & \\
\hline
\end{tabular}

NCCL+: subjects with non-carious cervical lesions. NCCL-: subjects without non-carious cervical lesions. $\left.{ }^{*}\right)$ Significant difference (Chi-square test, $\left.p<0.05\right)$

Table 2 Bivariate and multivariate regression analysis of the questionnaire variables

\begin{tabular}{|c|c|c|c|c|}
\hline Variables & PR (95\% Cl) & $p$-value & Adjusted PR (95\% Cl) & $p$-value \\
\hline \multicolumn{5}{|c|}{ Years of a sports activity } \\
\hline Up to 2 years & $1.09(0.5-2.37)$ & 0.835 & $2.74(0.97-7.72)$ & 0.056 \\
\hline More than 2 years & 1 & & 1 & \\
\hline \multicolumn{5}{|l|}{ Daily training time } \\
\hline Up to $1 \mathrm{~h}$ & $2.32(1.05-5.11)$ & $0.038^{*}$ & $3.35(1.18-9.49)$ & $0.023^{*}$ \\
\hline More than $1 \mathrm{~h}$ & 1 & & 1 & \\
\hline \multicolumn{5}{|l|}{ Toothpaste } \\
\hline Whitening & $1.29(0.49-3)$ & 0.684 & $1.64(0.56-4.79)$ & 0.369 \\
\hline Desensitizing & $1.61(0.65-4)$ & 0.304 & $5.26(1.63-16.96)$ & $0.004^{*}$ \\
\hline Conventional & 1 & & 1 & \\
\hline \multicolumn{5}{|l|}{ Medication } \\
\hline Yes & $0.5(0.14-1.75)$ & 0.278 & $0.5(0.19-1.27)$ & 0.144 \\
\hline No & 1 & & 1 & \\
\hline \multicolumn{5}{|c|}{ Lemon water intake while fasting } \\
\hline Yes & $2.5(1.67-3.75)$ & $<0.001^{*}$ & $8.1(2.19-29.9)$ & $0.002^{*}$ \\
\hline No & 1 & & 1 & \\
\hline \multicolumn{5}{|l|}{ Tooth sensitivity } \\
\hline Yes & $0.73(0.34-1.57)$ & 0.427 & $2.53(1.31-4.88)$ & $0.006^{*}$ \\
\hline No & 1 & & 1 & \\
\hline \multicolumn{5}{|c|}{ Previous orthodontic treatment } \\
\hline Yes & $1.58(0.77-3)$ & 0.231 & $3.11(1.48-6.5)$ & $0.003^{*}$ \\
\hline No & 1 & & 1 & \\
\hline \multicolumn{5}{|l|}{ Malocclusion } \\
\hline Angle Class III & $1.48(0.61-3.59)$ & 0.391 & $0.68(0.26-1.73)$ & 0.415 \\
\hline Angle Class II & $0.55(0.10-3.16)$ & 0.506 & $0.13(0.03-0.59)$ & $0.008^{*}$ \\
\hline Angle Class I & 1 & & 1 & \\
\hline \multicolumn{5}{|l|}{ Bite alteration } \\
\hline Yes & $1.1(0.54-2.22)$ & 0.795 & $1.53(0.77-3.07)$ & 0.226 \\
\hline No & 1 & & 1 & \\
\hline
\end{tabular}

PR prevalence ratio, $C l$ confidence interval. (*) Significant difference (Poisson Regression model, $p<0.05$ ) 
Table 3 Frequencies and percentages of the intraoral examination variables

\begin{tabular}{llllllllll}
\hline & Teeth & Occlusal wear & VAS tactile $(+)$ & VAS air $(+)$ & NCCLs $(+)$ & TWI & TWl & TWI & Class V restoration \\
& & & & & & 1 & 2 & 3 & 0 \\
\hline Incisors & $343(29.4 \%)$ & $136(39.7 \%)$ & $72(21 \%)$ & $84(24.5 \%)$ & $4(1.2 \%)$ & $4(1.2 \%)$ & $0(0 \%)$ & $0(0 \%)$ & $4(1.2 \%)$ \\
Canines & $172(14.7 \%)$ & $50(29.1 \%)$ & $42(24.4 \%)$ & $40(23.3 \%)$ & $12(7 \%)$ & $12(7 \%)$ & $0(0 \%)$ & $0(0 \%)$ & $1(0.3 \%)$ \\
Premolars & $331(28.4 \%)$ & $54(16.3 \%)$ & $60(18.1 \%)$ & $70(21.1 \%)$ & $56(16.9 \%)$ & $53(16 \%)$ & $3(0.9 \%)$ & $0(0 \%)$ & $0(0 \%)$ \\
Molars & $321(27.5 \%)$ & $35(10.9 \%)$ & $54(16.8 \%)$ & $50(15.6 \%)$ & $22(6.8 \%)$ & $19(5.9 \%)$ & $3(0.9 \%)$ & $0(0 \%)$ & $8(2 \%)$ \\
Upper & $585(50.1 \%)$ & $119(20.3 \%)$ & $103(17.6 \%)$ & $111(19 \%)$ & $51(8.8 \%)$ & $46(7.9 \%)$ & $5(0.9 \%)$ & $0(0 \%)$ & $7(1.2 \%)$ \\
Lower & $582(49.9 \%)$ & $156(26.8 \%)$ & $125(21.5 \%)$ & $133(22.9 \%)$ & $43(7.4 \%)$ & $42(7.2 \%)$ & $1(0.2 \%)$ & $0(0 \%)$ & $6(1 \%)$ \\
Right & $584(50 \%)$ & $140(24.0 \%)$ & $116(19.9 \%)$ & $107(18.3 \%)$ & $58(9.9 \%)$ & $54(9.2 \%)$ & $4(0.7 \%)$ & $0(0 \%)$ & $9(1.5 \%)$ \\
Left & $583(50 \%)$ & $135(23.1 \%)$ & $112(19.2 \%)$ & $137(23.5 \%)$ & $36(6.1 \%)$ & $34(5.8 \%)$ & $2(0.3 \%)$ & $0(0 \%)$ & $4(0.7 \%)$ \\
Total & $1167(100 \%)$ & $275(23.6 \%)$ & $228(19.5 \%)$ & $244(20.9 \%)$ & $94(8 \%)$ & $88(7.5 \%)$ & $6(0.5 \%)$ & $0(0 \%)$ & $13(1.1 \%)$ \\
\hline
\end{tabular}

without sclerotic dentin (98\%), and smooth texture (99\%). Figure 1 shows the morphological aspects of some NCCLs.

The laboratory assays of participants with or without NCCLs revealed regular salivary flow (higher than $0.7 \mathrm{ml} / \mathrm{min}$ ), neutral $\mathrm{pH}$, and buffering capacity above 5.5. All participants presented similar amounts of $\mathrm{Ca}, \mathrm{Na}$, and $\mathrm{K}$ ions, and their level of cortisol remained bellow $20.3 \mathrm{nmol} / \mathrm{l}$. Therefore, none of the salivary analyses indicated significant differences.

\section{Discussion}

This study diagnosed a remarkable prevalence of NCCLs in footballers and revealed the daily training time as an important risk indicator. The NCCLs were diagnosed in $39.5 \%$ of the participants, while the data in the literature varies from 5 to $85 \%$ due to different types of participants (specific subgroups of the general population) combined with the lack of method standardization [2, 16]. For instance, a study involving Brazilians between 40 to 60 years old reported NCCLs prevalence of $67 \%$ while a population from 35 to 74 years old in China presented a prevalence of $81.3 \%[17,18]$. However, a recent clinical trial with patients between 18 and 40 years old

Table 4 Relationship between occlusal/incisal tooth wear and NCCL depth

\begin{tabular}{|c|c|c|c|c|c|}
\hline & \multicolumn{4}{|l|}{ NCCL depth } & \multirow{2}{*}{$\begin{array}{l}p- \\
\text { value }\end{array}$} \\
\hline & 0 & $<1 \mathrm{~mm}$ & $1-2 \mathrm{~mm}$ & Total & \\
\hline \multicolumn{6}{|c|}{$\begin{array}{l}\text { Occlusal/incisal } \\
\text { tooth wear }\end{array}$} \\
\hline 0 & $802(75.6 \%)$ & 73 (83\%) & $6(100 \%)$ & 881 (76.3\%) & 0.077 \\
\hline 1 & $213(20.1 \%)$ & 15 (17\%) & $0(0 \%)$ & 228 (19.7\%) & \\
\hline 2 & 41 (3.9\%) & $0(0 \%)$ & $0(0 \%)$ & 41 (3.6\%) & \\
\hline 3 & $4(0.4 \%)$ & $0(0 \%)$ & $0(0 \%)$ & $4(0.3 \%)$ & \\
\hline Total & 1060 (100\%) & 88 (100\%) & $6(100 \%)$ & 1154 (100\%) & \\
\hline
\end{tabular}

(*) Significant difference (Chi-square test, $p \leq 0.05)$ treated for NCCLs and cervical dentin hypersensitivity at a Brazilian university revealed a prevalence of $88.1 \%$ and also suggested an increasing distribution of NCCLs among youths [1]. Other studies reported prevalence ratios below 53\% [19-23].

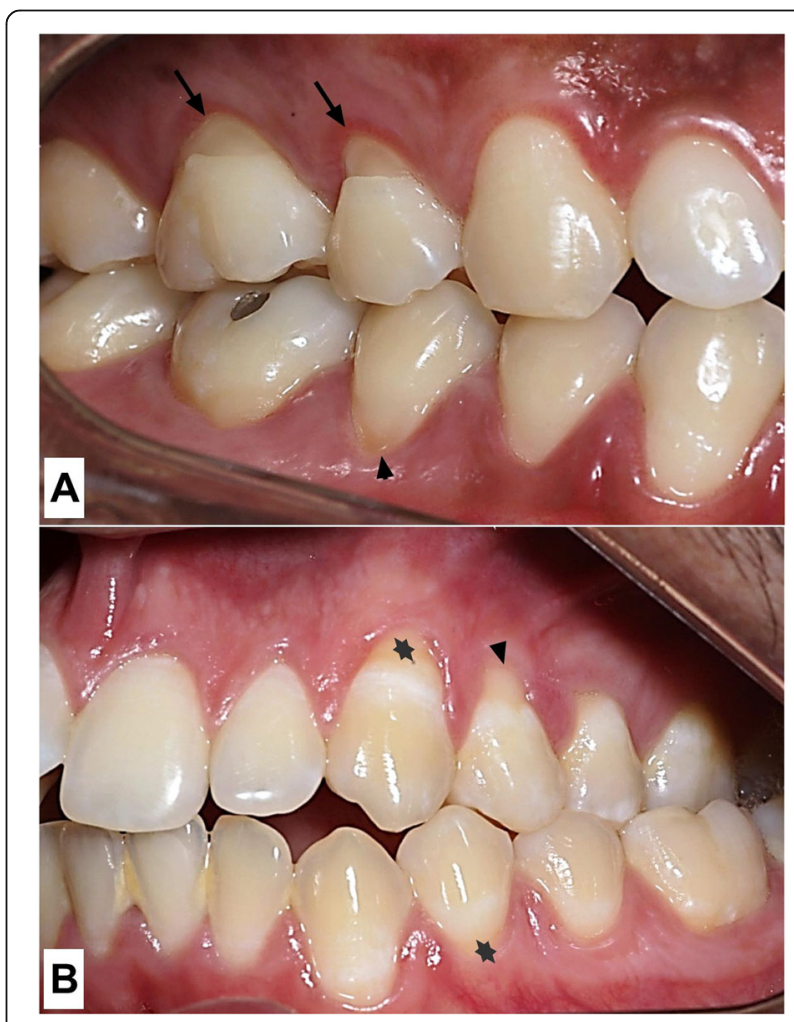

Fig. 1 Clinical appearance of NCCLs. a The upper black arrows indicates wedge-shaped moderate NCCLs (1-2 mm depth) while the lower black arrowhead shows a rounded shallow NCCL $(<1 \mathrm{~mm}$ depth). All lesions are located coronally to the CEJ, present supragingival clinical location, absence of sclerotic dentin and smooth texture. $\mathbf{b}$ White spots at the cervical teeth outline. The asterisks highlights the white spots (suggestive of cracks) that do not extend to the root in case of gingival recession (black arrowhead) 
The bivariate regression analysis revealed that daily training time is a dominant risk indicator for the development of NCCLs. The fact that footballers that reported only up to $1 \mathrm{~h}$ of daily training presented more NCCLs may be related to poor physical conditioning. Therefore, the footballers that trained more than $1 \mathrm{~h}$ per day seem to better withstand the intensity and directions of the loads that teeth are subjected to. Similarly, Antunes et al. observed that the training time of Brazilian amateur runners represented a risk factor for dental erosion [22]. Approximately $52 \%$ of the runners that expended only up to $1 \mathrm{~h}$ during training presented tooth erosion, while the rate decreased to 38 and $9.5 \%$ for those runners that trained up to 2 and $3 \mathrm{~h}$, respectively. In addition to good physical conditioning, individuals dedicated to long training times possibly take better healthcare. The intake of lemon water while fasting was significantly associated with NCCLs. Acids of either exogenous (some fruits and juices, carbonated soft drinks, acidic sports beverages, alcoholic drinks, some medications, and occupational factors such as chlorine used in swimming pools) or endogenous origin (gastric juice) contribute to dental erosion and consequently to the development of NCCLs [24-29].

The multivariate regression analysis significantly associated the prevalence of NCCLs with several variables (daily training time, lemon water intake while fasting, use of desensitizing toothpaste, tooth sensitivity, and previous orthodontic treatment), which confirms that the combination of factors is more relevant in the development of these lesions $[1,7]$.

Our findings reinforce that the indiscriminate use of desensitizing toothpaste without professional follow-up impairs the early diagnosis of NCCLs since the painful symptoms are masked and the individual does not seek appropriate treatment. The presence of tooth sensitivity allows proper NCCL diagnosis and its association with other variables favors the development of the lesions [30].

Although orthodontic treatment induces stress in the cervical region of teeth, the intensity of the force applied by orthodontic appliances should not exceed the intrinsic tensile and compression strength of dental structures [31, 32]. Spini assessed the cervical tension of premolars through finite element analysis and extensiometry and concluded that the accumulation of tension and deformity was higher in teeth with NCCL and previously subjected to orthodontics [33]. In our study, the significant association between orthodontic treatment and NCCLs may be explained by the excessive forces that teeth were subjected during successive years and caused structural losses of enamel and alveolar bone. The increasing prevalence of NCCLs among youths represents novel information to the literature. However, it was observed that most participants did not have their orthodontic treatment completed.

Brandini et al. [19] and Kolak et al. [20] evaluated men and women with a similar age range and diagnosed NCCLs in 13.2 and $3.1 \%$ of teeth, respectively. In comparison with the abovementioned studies, our prevalence of NCCLs in the examined teeth remained in the intermediate percentage level of $8 \%$ and the lesions were more frequently found in upper premolars. It is a consensus in the literature that premolars present the highest prevalence of NCCLs due to an unfavorable crown-root proportion and the thin buccal bone plate. These features result in a highstress concentration in the cervical region during eccentric mandibular movements, particularly in the case of group disocclusion [1, 17, 19, 20, 34-37].

The diagnosed NCCLs in this study were predominantly shallow, rounded, supragingival, and without sclerotic dentin, which characterizes them as initial lesions [38]. However, many participants also presented white spots at the cervical teeth outline that does not extend to the root in case of gingival recession. The white color does not change whether the enamel is wet or dry and the concentration varies from diffuse to a well-defined horizontal line. Considering that the footballers are constantly subjected to either tension or mechanical stress, we diagnosed these spots as cracks. This is supported by the concept of striations, which were defined as irregular horizontal enamel bands in the cervical region affected by molecular decomposition (molecular slips or Luder lines) [39]. Lee and Eakles [40] stated that when hydroxyapatite crystals are ruptured, the spaces are filled with water molecules from saliva that impair chemical bonds between crystals and the tooth becomes more vulnerable to mechanical and erosive damages. The water penetration into the dental structure may explain such a whitish aspect and the different shades may be related to the crack depth.

Posterior teeth showed the highest and lowest frequencies of tooth sensitivity and occlusal wear, respectively. Interestingly, the opposite trend was observed for incisors and canines; however, no correlation was found. The different number of participants with or without NCCLs figures as a limitation of this study that reduces the statistical efficiency.

The relationship between the occlusal/incisal wear and NCCL depth was inverse. Among the 94 teeth with NCCLs, 79 did not present occlusal/incisal wear, and 15 presented exclusive enamel wear. Senna et al. [41] conducted a systematic review on the association between NCCLs and occlusion and suggested that occlusal wear is a nature's way to eliminate occlusal disturbances. The authors also reported that NCCLs develop before or during the establishment of occlusal wear when the teeth are subjected to occlusal stress. Despite the role of 
tension in the development of NCCLs, an inversive relationship was observed in our study since footballers with NCCLs showed little or no occlusal/incisal wear.

It was observed that the incidence of positive sensitivity in premolars (18.1 to $21.1 \%$ ) was higher than the incidence of NCCLs (16.9\%). We concluded that tooth sensitivity combined with the presence of white spots (cracks induced by stress) in many participants is symptoms/signs of initial NCCL.

The laboratory assays indicated normal salivary parameters in those participants with NCCLs; therefore, these lesions seem related to excessive mechanical stress and not to erosive factors such as acidic beverages or brushing immediately after meals. Fullagar et al. reported that footballers possess limited nutrition and hydration practices [42]. The level of cortisol of all participants was found normal. Although cortisol is regarded as a stress biomarker, the level of anxiety in footballers varies greatly depending on their physical performance [43-45]. After evaluating both trained and untrained men, Rimmele et al. reported that physical activity may provide a protective effect against disorders related to stress since it regulates the level of cortisol [46].

Despite the symptoms/signs of initial NCCLs, the examined footballers need preventive or even curative treatment to control the progression of the lesions. Moreover, periodic follow-ups of their salivary and nutritional conditions are important to dental health. Due regard must be given to the daily training time as an important risk indicator of NCCLs development since the contemporary lifestyle of youths includes intensive physical exercises and frequent intake of acidic sports beverages. Therefore, clinicians must investigate and guide patients to reduce the risk of NCCLs development.

\section{Conclusion}

The prevalence of NCCLs among footballers was remarkable. The premolars were the most affected teeth and presented symptoms/signs of initial lesions. The daily training time was a dominant risk indicator of NCCLs development. These lesions were also significantly associated with several indicators (daily training time, lemon water intake while fasting, use of desensitizing toothpaste, tooth sensitivity, and previous orthodontic treatment). Footballers presented adequate salivary parameters and cortisol levels.

\section{Supplementary information}

Supplementary information accompanies this paper at https://doi.org/10. 1186/s12903-020-01200-9.

Additional file 1: Supplementary file: Questionnaire of

socioeconomic status, medical history and habits. The questionnaire has been developed for this study.

\section{Abbreviations}

NCCLs: Non-carious cervical lesions; Ca: Calcium; Na: Aodium; K: Potassium; CEJ: Cementoenamel junction; TMJ: Temporomandibular joint; TWI: Tooth wear index; mm: Millimeter; psi: Pound per square inch; VAS: Visual analog scale; nmol/l: Nanomoles per liter; min: Minutes; ml: Milliliter; ICPOES: Inductively-coupled plasma optical emission spectrometer;

PR: Prevalence ratio; Cl: Confidence interval

\section{Acknowledgements}

The authors thank FAPESPA - Amazonia Foundation for the Support of Studies and Research, PhD David Normando and PhD Rafael Lima for supporting this study.

\section{Authors' contributions}

T L M M contributed to the acquisition, analysis, interpretation of data, and was a major contributor in writing the manuscript. S C A N M contributed to the acquisition and analysis of patient data. D G E contributed to the acquisition analysis of patient image data. K C F F contributed to the acquisition analysis of laboratory data on patients. H H C P contributed to the analysis, interpretation of statistical data on patients and substantively revised the manuscript. R S D C contributed to the design of the work, analysis, interpretation the all data and substantively revised the manuscript. All authors read and approved the final manuscript.

\section{Funding}

This research was supported by Pró-Reitoria de Pesquisa e Pós Graduação da UFPA (PROPESP/UFPA, Brazil).

\section{Availability of data and materials}

The datasets used and/or analysed during the current study are available from the corresponding author on reasonable request.

\section{Ethics approval and consent to participate}

The research involved human participants, human material, human data and was performed in accordance with the Declaration of Helsinki. Our study was approved by the Ethics Committee of the Federal University of Pará (CEP/ICS-UFPA) - protocol number CAEE: (86808318.2.0000.0018).

A written informed consent form was voluntarily signed by all participants.

\section{Competing interests}

The authors declare that there is no conflict of interest regarding the publication of this article.

\section{Author details}

${ }^{1}$ School of Dentistry, Federal University of Pará, Augusto Corrêa Street 937 odd side, Guamá, Belém, PA 66075110, Brazil. Evandro Chagas Institute, BR316 - km7, Marituba, Pará, Brazil.

Received: 23 December 2019 Accepted: 20 July 2020

Published online: 29 July 2020

\section{References}

1. Teixeira DNR, Zeola LF, Machado AC, Gomes RR, Souza PG, Mendes DC, et al. Relationship between noncarious cervical lesions, cervical dentin hypersensitivity, gingival recession, and associated risk factors: a crosssectional study. J Dent. 2018;76:93-7.

2. Levitch LC, Bader JD, Shugars DA, Heymann HO. Non-carious cervical lesions. J Dent. 1994;22:195-207.

3. Bader JD, McClure F, Scurria MS, Shugars DA, Heymann HO. Case-control study of non-carious cervical lesions. Community Dent Oral Epidemiol. 1996; 24:286-91.

4. Hur B, Kim HC, Park JK, Versluis A. Characteristics of non-carious cervical lesions - an ex vivo study using micro computed tomography. J Oral. Rehabil. 2011:38:469-74.

5. Wada I, Shimada Y, Ikeda M, Sadr A, Nakashima S, Tagami J, et al. Clinical assessment of non carious cervical lesion using swept-source optical coherence tomography. J Biophotonics. 2015;8:846-54.

6. Walter $\mathrm{C}$, Kress E, Götz H, Taylor K, Willershausen I, Zampelis A. The anatomy of non-carious cervical lesions. Clin Oral Investig. 2014;18:139-46. 
7. Grippo JO, Simring M, Coleman TA. Abfraction, abrasion, biocorrosion, and the enigma of noncarious cervical lesions: a 20-year perspective. J Esthet Restor Dent. 2012;24:10-23.

8. Frese C, Frese F, Kuhlmann S, Saure D, Reljic D, Staehle HJ, et al. Effect of endurance training on dental erosion, caries, and saliva. Scand J Med Sci Sport. 2015;25:e319-26.

9. Broad EM, Rye LA. Do current sports nutrition guidelines conflict with good oral health? Gen Dent. 2015;63:18-23.

10. Frese C, Wohlrab T, Sheng L, Kieser M, Krisam J, Frese F, et al. Clinical management and prevention of dental caries in athletes: a four-year randomized controlled clinical trial. Sci Rep. 2018;8:16991.

11. Soares PV, Grippo JO. Noncarious cervical lesions and cervical dentin hypersensitivity: etiology, diagnosis and treatment. 1st ed. Chicago: Quintessence Publishing Co; 2017.

12. Vieira EP, Barbosa MS, Quintão CC, Normando D. Relationship of tooth wear to chronological age among indigenous Amazon populations. PLoS One. 2015:10:e0116138.

13. Angle EH. Classification of malocclusion. Dent Cosm. 1899;41:248-64.

14. Thylstrup A, Fejerskov O. Clinical and pathological features of dental caries. In: Weyne S, Oppermann R, editors. Textbook of clinical cariology. 2nd ed. São Paulo: Santos: 1995. p. 111-57.

15. Krasse B. Risco de Cárie. 2nd ed. São Paulo: Quintessence; 1998.

16. Favaro-Zeola L, Soares PV, Cunha-Cruz J. Prevalence of dentin hypersensitivity: systematic review and meta-analysis. J Dent. 2019;81:1-6.

17. Smith WA, Marchan S, Rafeek RN. The prevalence and severity of noncarious cervical lesions in a group of patients attending a university hospital in Trinidad. J Oral Rehabil. 2008;35:128-34.

18. Lai ZY, Zhi QH, Zhou Y, Lin HC. Prevalence of non-carious cervical lesions and associated risk indicators in middle-aged and elderly populations in southern China. Chin J Dent Res. 2015:18:41-50.

19. Brandini DA, Trevisan $C L$, Panzarini SR, Pedrini D. Clinical evaluation of the association between noncarious cervical lesions and occlusal forces. J Prosthet Dent. 2012;108:298-303.

20. Kolak V, Pešić D, Melih I, Lalović M, Nikitović A, Jakovljević A. Epidemiological investigation of non-carious cervical lesions and possible etiological factors. J Clin Exp Dent. 2018;10:e648-56.

21. Buczkowska-Radlińska J, Łagocka R, Kaczmarek K, Górski M, Nowicka A. Prevalence of dental erosion in adolescent competitive swimmers exposed to gas-chlorinated swimming pool water. Clin Oral Invest. 2012;17:579-83.

22. Antunes LS, Veiga L, Nery VS, Nery CC, Antunes LA. Sports drink consumption and dental erosion among amateur runners. J Oral Sci. 2017;59:639-43.

23. Zuza A, Racic M, Ivkovic N, Krunic J, Stojanovic N, Bozovic D, et al. Prevalence of non-carious cervical lesions among the general population of the republic of Srpska, Bosnia and Herzegovina. Int Dent J. 2019;69:281-8.

24. Dawes C. Physiological factors affecting salivary flow rate, oral sugar clearance, and the sensation of dry mouth in man. J Dent Res. 1987;66:648-53.

25. Barron RP, Carmichael RP, Marcon MA, Sàndor GK. Dental erosion in gastroesophageal reflux disease. J Can Dent Assoc. 2003;69:84-9.

26. Hara ES, Matsuka Y, Minakuchi H, Clark GT, Kuboki T. Occlusal dysesthesia: a qualitative systematic review of the epidemiology, a etiology and management. J Oral Rehabil. 2012;39:630-8.

27. Buzalaf MAR, Magalhães AC, Rios D. Prevention of erosive tooth wear: targeting nutritional and patient-related risks factors. Br Dent J. 2018;224: 371-8.

28. Schlueter N, Luka B. Erosive tooth wear - a review on global prevalence and on its prevalence in risk groups. Br Dent J. 2018;224:364-70.

29. Alcântara PM, Barroso NFF, Botelho AM, Douglas-de-Oliveira DW, Gonçalves PF, Flecha OD. Associated factors to cervical dentin hypersensitivity in adults: a transversal study. BMC Oral Health. 2018;18:155.

30. Que K, Guo B, Jia Z, Chen Z, Yang J, Gao P. A cross-sectional study: noncarious cervical lesions, cervical dentine hypersensitivity and related risk factors. J Oral Rehabil. 2013:40:24-32

31. Rees JS. The effect of variation in occlusal loading on the development of abfraction lesions: a finite element study. J Oral Rehabil. 2002;29:188-93.

32. Jakupovič S, Anič I, Ajanovič M, Korać S, Konjhodžić A, Džanković A, et al Biomechanics of cervical tooth region and noncarious cervical lesions of different morphology; three-dimensional finite element analysis. Eur J Dent. 2016:10:413-8

33. Spini, PHR: Effect of orthodontic movements on the biomechanical behavior of premolars with noncarious cervical lesions. https://repositorio. ufu.br/handle/123456789/17035 (2016). Acessed 15 Dec 2019
34. Pegoraro LF, Scolaro JM, Conti PC, Telles D, Pegoraro TA. Noncarious cervical lesions in adults: prevalence and occlusal aspects. J Am Dent Assoc. 2005;136:1694-700.

35. Palomino-Gómez SP, Jeremias F, Finoti LS, Paredes-Coz G, Raveli DB. Influence of lateral excursion on vestibular cervical dental abfraction. Acta Odontol Latinoam. 2011;24:283-8.

36. Soares PV, Souza LV, Veríssimo C, Zeola LF, Pereira AG, Santos-Filho PCF et al. Effect of root morphology on biomechanical behaviour of premolars associated with abfraction lesions and different loading types. J Oral Rehabil. 2014;41:108-14.

37. Igarashi Y, Yoshida S, Kanazawa E. The prevalence and morphological types of non-carious cervical lesions (NCCL) in a contemporary sample of people. Odontology. 2017;105:443-52.

38. Michael JA, Kaidonis JA, Townsend GC. Non-carious cervical lesions on permanent anterior teeth: a new morphological classification. Aust Dent J. 2010;55:134-7.

39. Grippo JO. Abfractions: a new classification of hard tissue lesions of teeth. J Esthet Dent. 1991:3:14-9.

40. Lee WC, Eakle WS. Stress-induced cervical lesions: review of advances in the past 10 years. J Prosthet Dent. 1996;75:487-94.

41. Senna P, Del Bel CA, Rösing C. Non-carious cervical lesions and occlusion: a systematic review of clinical studies. J Oral Rehabil. 2012:39:450-62.

42. Fullagar HHK, McCunn R, Murray A. Updated review of the applied physiology of American college football: physical demands, strength and conditioning, nutrition, and injury characteristics of america's favorite game. Int J Sports Physiol Perform. 2017:12:1396-403.

43. Brenner I, Shek PN, Zamecnik J, Shephard RJ. Stress hormones and the immunological responses to heat and exercise. Int J Sports Med. 1998;19:130-43.

44. Hanton S, Thomas O, Maynard I. Competitive anxiety responses in the week leading up to competition: the role of intensity, direction and frequency dimensions. Psychol Sport Exerc. 2004;5:169-81.

45. Filaire E, Alix D, Ferrand C, Verger M. Psychophysiological stress in tennis players during the first single match of a tournament. Psychoneuroendocrinology. 2009;34:150-7.

46. Rimmele U, Zellweger BC, Marti B, Seiler R, Mohiyeddini C, Ehlert U, et al. Trained men show lower cortisol, heart rate and psychological responses to psychosocial stress compared with untrained men.

Psychoneuroendocrinology. 2007;32:627-35.

\section{Publisher's Note}

Springer Nature remains neutral with regard to jurisdictional claims in published maps and institutional affiliations.
Ready to submit your research? Choose BMC and benefit from:

- fast, convenient online submission

- thorough peer review by experienced researchers in your field

- rapid publication on acceptance

- support for research data, including large and complex data types

- gold Open Access which fosters wider collaboration and increased citations

- maximum visibility for your research: over $100 \mathrm{M}$ website views per year

At BMC, research is always in progress.

Learn more biomedcentral.com/submissions 